\title{
On Smoothing Surfaces in Voxel Based Finite Element Analysis of Trabecular Bone
}

\author{
Peter Arbenz and Cyril Flaig \\ Institute of Computational Science, ETH Zürich, CH-8092 Zürich
}

\begin{abstract}
The (micro-)finite element analysis based on three-dimensional computed tomography (CT) data of human bone takes place on complicated domains composed of often hundreds of millions of voxel elements. The finite element analysis is used to determine stresses and strains at the trabecular level of bone. It is even used to predict fracture of osteoporotic bone. However, the computed stresses can deteriorate at the jagged surface of the voxel model.

There are algorithms known to smooth surfaces of voxel models. Smoothing however can distort the element geometries. In this study we investigate the effects of smoothing on the accuracy of the finite element solution, on the condition of the resulting system matrix, and on the effectiveness of the smoothed aggregation multigrid preconditioned conjugate gradient method.
\end{abstract}

\section{Introduction}

In view of the growing importance of osteoporosis due to the obsolescence of the population in industrialized countries an accurate analysis of individual bone strength is in dire need. In fact according to the WHO, lifetime risk for osteoporotic fractures in women is estimated close to $40 \%$; in men risk is $13 \%$ [7. With the advent of fast and powerful computers, simulation techniques are becoming popular for investigating the mechanical properties of bones and predicting the strength of a given patient's bones. In order to gain an improved comprehension of structure and strength of bone, large scale computer simulations are executed based on the theory of (non)linear elasticity and the finite element method.

Today's approach is based on three-dimensional computed tomography $(\mathrm{CT})$ whereby bones are scanned with a resolution of $50-100 \mu \mathrm{m}$. Using a direct voxelconversion technique the three-dimensional computer reconstructions of bone can be converted to a finite element mesh, that can be used to perform a 'virtual experiment', i.e., to simulate a mechanical test in great detail and with high precision. The resulting procedure is called microstructural finite element $(\mu \mathrm{FE})$ analysis.

The approach based on the FE analysis leads to linear systems of equations

$$
K \mathbf{u}=\mathbf{f},
$$

where the stiffness matrix $K$ is symmetric positive-definite, the components of the vector $\mathbf{u}$ are the displacements at the nodes of the voxel mesh. $\mathbf{f}$ contains external loads or prescribed displacements.

I. Lirkov, S. Margenov, and J. Waśniewski (Eds.): LSSC 2007, LNCS 4818, pp. 6977 2008. (C) Springer-Verlag Berlin Heidelberg 2008 
The system of equations (11) can be solved very efficiently by the conjugate gradient algorithm preconditioned by smoothed aggregation multigrid. Systems of up to hundreds of millions of degrees of freedom have been solved on large scale computers within a couple of minutes [1,2,3].

The voxel approach has deficiencies though. In particular, the jagged domains leading to exceeding stresses at the nodes of the mesh corresponding to corners of the domain. A straightforward procedure is to smooth the surface of the computational domain. Taubin [9,10] suggested a surface fairing algorithm that does not shrink the body it embraces. Boyd and Müller [4 have applied this algorithm to voxel based models. In this note we investigate this latter algorithm in a parallel environment. In section 2 we discuss how smoothing can be done with piecewise trilinear isoparametric hexahedral elements. In section 3 we discuss the effects of the flexible elements on visualization, stresses, and condition of the stiffness matrix. We also mention how the computational work can be reduced by splitting distorted hexahedra in piecewise linear tetrahedral elements.

\section{Smoothing}

In bone structure analysis the computational domain is composed of a multitude of tiny cubes, so-called voxels, that are generated directly from the output of the CT scanner. Surface patches and edges are always aligned with the coordinate directions. In contrast to the originally smooth object, the voxel model has a jagged surface. The stresses induced by the computed displacements can have singularities at edges and corners of the surface but also of interfaces between different materials. A straightforward approach to get rid of the singular stresses is to smooth the surface and material interfaces of the computational domain.

In computer graphics there is a well-known procedure to smooth polygonal surfaces called mesh fairing [9,10]. The coordinates $\mathbf{x}$ of the mesh vertices are moved according to the diffusion equation

$$
\frac{\partial \mathbf{x}}{\partial t}=D \cdot \Delta \mathbf{x}, \quad D>0,
$$

where the Laplacian at $\mathbf{x}$ is approximated by

$$
\Delta \mathbf{x}_{i}=\sum_{j \in \mathfrak{N}(i)} w_{i j}\left(\mathbf{x}_{j}-\mathbf{x}_{i}\right), \quad \sum_{j \in \mathfrak{N}(i)} w_{i j}=1 .
$$

Here, $\mathfrak{N}(i)$ denotes the neighbor nodes of node $i$. The choice of the weights evidently affects the quality of the smoothing 10 . The most effective scheme is due to Fujiwara, where $w_{i j}$ is proportional to $1 /\left\|\mathbf{x}_{j}-\mathbf{x}_{i}\right\|$.

Applying Euler's method with time step $\Delta t$ to (2) we get

$$
\mathbf{x}_{i}^{\text {new }}=\mathbf{x}_{i}^{\text {old }}+\lambda \Delta \mathbf{x}_{i}^{\text {old }}, \quad \lambda=D \Delta t .
$$

Boyd and Müller [4] applied this technique to the voxel model by classifying nodes as fixed nodes, surface nodes, interface nodes, inner nodes and near-surface 
nodes. Fixed and inner nodes must not move; this is accomplished by leaving empty their set of neighbors. Surface and interface nodes produce a surface mesh which is smoothed by the algorithm. Since only surface and interface nodes can qualify as neighbors of surface and interface nodes, these nodes are subjected to Taubin's original 2D algorithm. Near-surface nodes should compensate for the movements of the surface and interface nodes, such that hexahedra near the surface are not seriously distorted.

The smoothing procedure (44) shrinks the volume of the 3D object it is applied to. To compensate this effect, Taubin suggests to replace $\lambda$ in every second step by $-\mu$ where $\mu=\lambda$ or slightly bigger [9]. The 'negative diffusion' has the effect of (approximately) restoring the volume of the 3D object.

This smoothing procedure was incorporated into our fully-parallel $\mu$-finite element code PARFE [ 8 that is based on the Trilinos framework [1], see [6] for details. A piecewise trilinear finite element space was implemented on the distorted mesh based on isoparametric hexahedral elements. The matrix elements have been computed approximately by the 64 -point tensor product Gaussian quadrature rule.

\section{$3 \quad$ Results}

In this section we discuss four effects of smoothing, (1) the visual quality, (2) the condition number of the stiffness matrix, (3) the scalability of the smoothing procedure, and (4) the cost of the assembling of the matrix. The computations have been done on the Cray XT3 at the Swiss National Supercomputer Center.

To show the visual quality of the smoothing algorithm a sphere was smoothed with $\lambda$ and $\mu$ as proposed in [10,4]. A sphere has an absolutely smooth surface to which the surface coordinates should converge. The smoothing procedure in fact generates quite a smooth surface, see Fig. 11. The implemented procedure
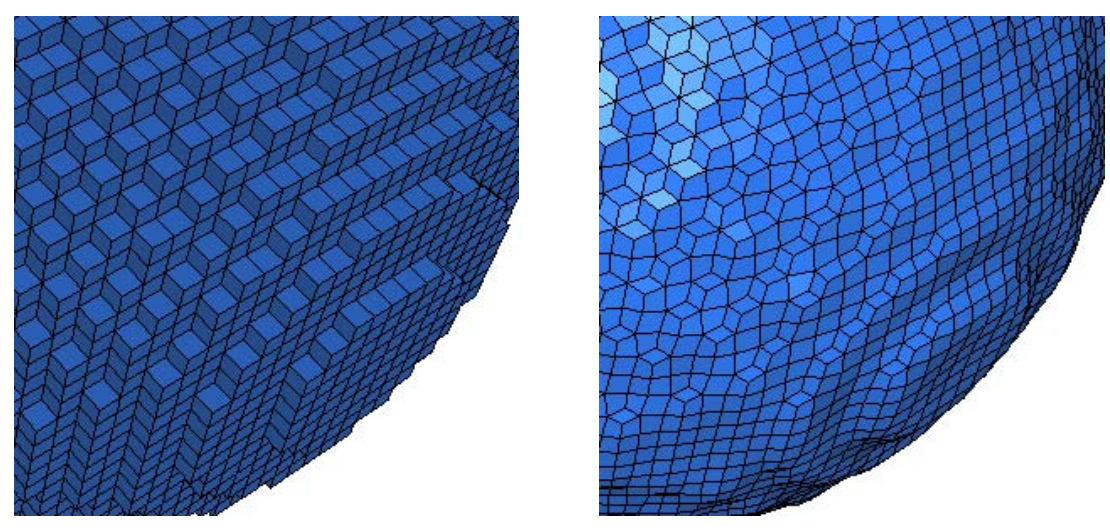

Fig. 1. A sample sphere. On the left is the original; on the right is a smoothed sphere subject to 32 smoothing steps with $\lambda=0.4$ and $1 / \mu+1 / \lambda=0.1$. 

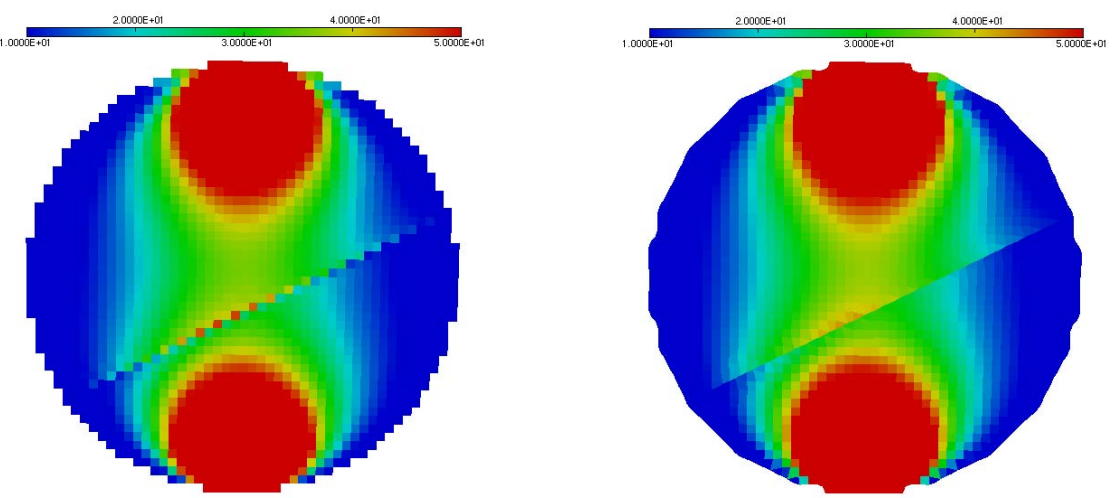

Fig. 2. A clip through a sphere consisting of two materials. The color indicates the stress. The original jagged sphere is on the left; the smoothed sphere is on the right after 32 smoothing steps with the parameters as in Fig. 1 The stresses do not oscillate so much in the smoothed version as in the unsmoothed version.

Table 1. Impact of the number of smoothing steps on maximal and mean stress

\begin{tabular}{|l|c|c|c|c|c|c|}
\hline & \multicolumn{3}{|c|}{ Sphere } & \multicolumn{3}{c|}{ cube1 } \\
\hline Smoothing steps & 0 & 8 & 16 & 0 & 8 & 16 \\
\hline Max stress (MPa) & 367.7 & 365.3 & 365.6 & 231.6 & 235.2 & 237.0 \\
\hline Mean stress (MPa) & 20.8 & 20.73 & 20.4 & 19.6 & 20.1 & 20.3 \\
\hline
\end{tabular}

not only smoothes surfaces, but also interfaces between differing materials, as can be seen from Fig. 2. To investigate how the visual impression changes as the number of smoothing steps increases we consider a bone specimen consisting of 98'381 voxels. From Fig. 3 we see that already very few smoothing steps lead to dramatically improved surfaces. After 28 steps, however, some voxels get so much distorted that the stiffness matrix loses definiteness. Also the visual impression does not improve much beyond this point. It is possible but to time consuming to check individual elements for strong distortion and detach them from the smoothing process.

The deformed hexahedra not only have an effect on the visual impression, but also change the distribution of the stresses. Boyd and Müller 4 report that the peak stresses are lowered by a factor 4 for a sphere model. Camacho et al. [5] describe a similar effect for the von Mises stresses.

We used two models to measure the stress: a sphere consisting of two materials and a bone sample (cube1 in [2]) consisting of one material. We applied a varying number of smoothing steps with $\lambda=0.4$. As suggested in [10, 4] we determined $\mu$ from $1 / \lambda+1 / \mu=0.1$. Both models are fixed at $z=0$ and a load is exerted on the top plane $\left(z=z_{\max }\right)$.

Table 1 shows the maximal and the mean von Mises stresses. The smoothing procedure has a minimal effect on these. The maximal stress is observed at the verge of the loading. Fig. 2 shows that the stresses propagate through the 


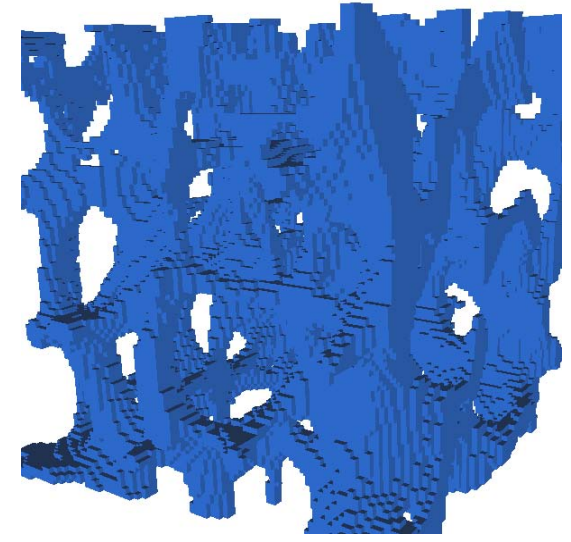

(a)

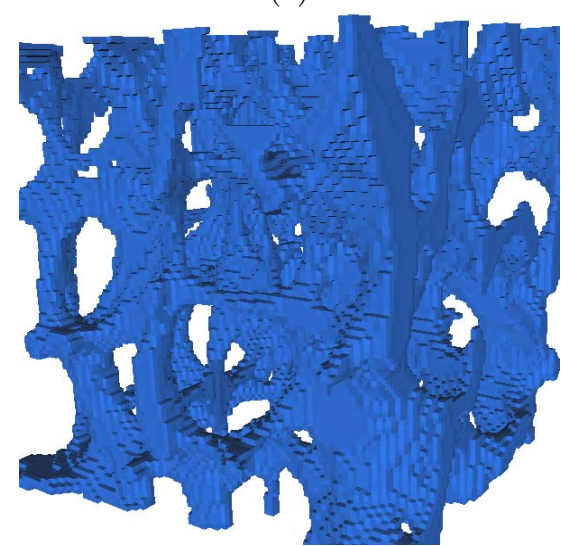

(c)

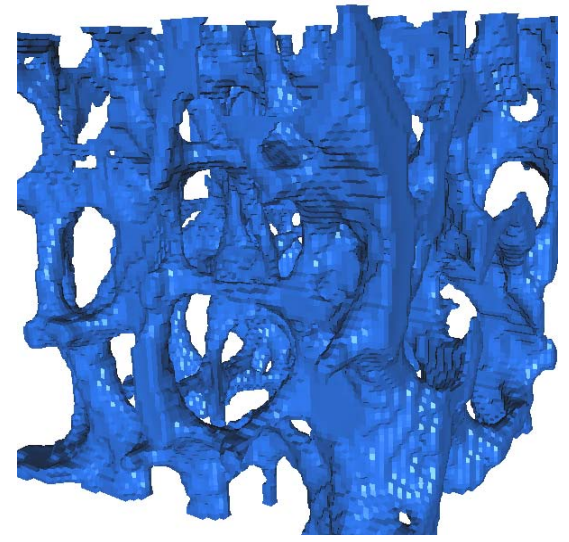

(e)

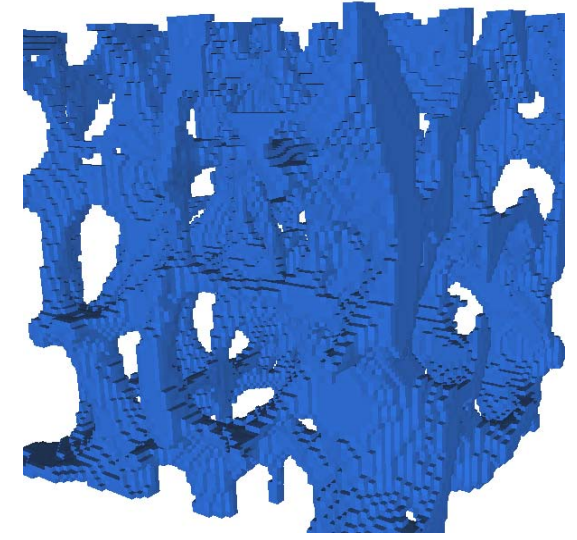

(b)

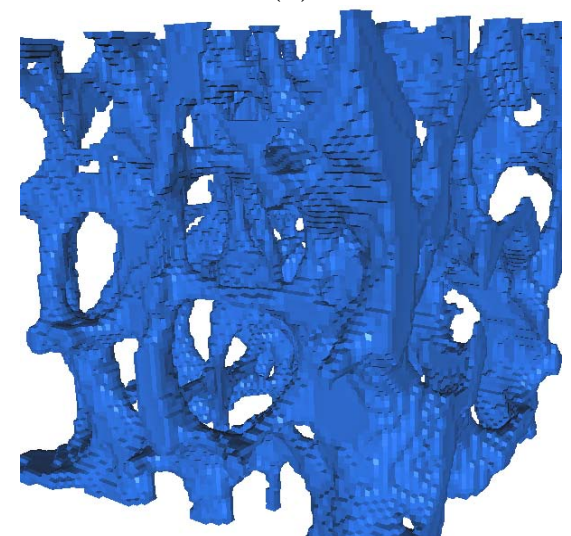

(d)

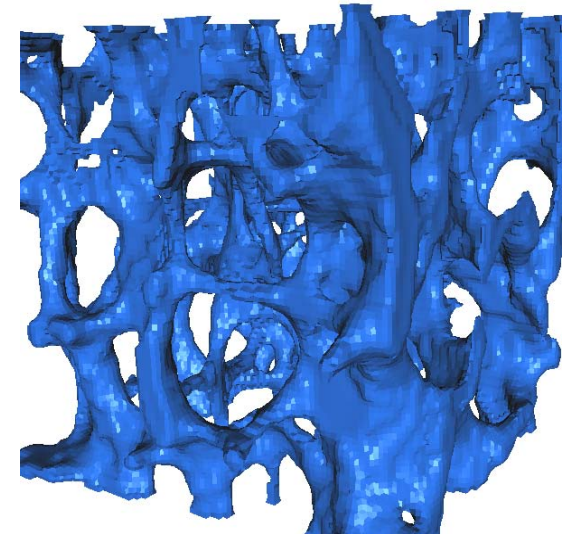

(f)

Fig. 3. A sample mesh representing trabecular bone. The unsmoothed mesh (a) was smoothed with 4 (b), 8 (c), 16 (d), 28 (e), and 64 (f) steps, respectively. The step size was 0.4 . With more than 28 smoothing steps of the deformations became too severe. 


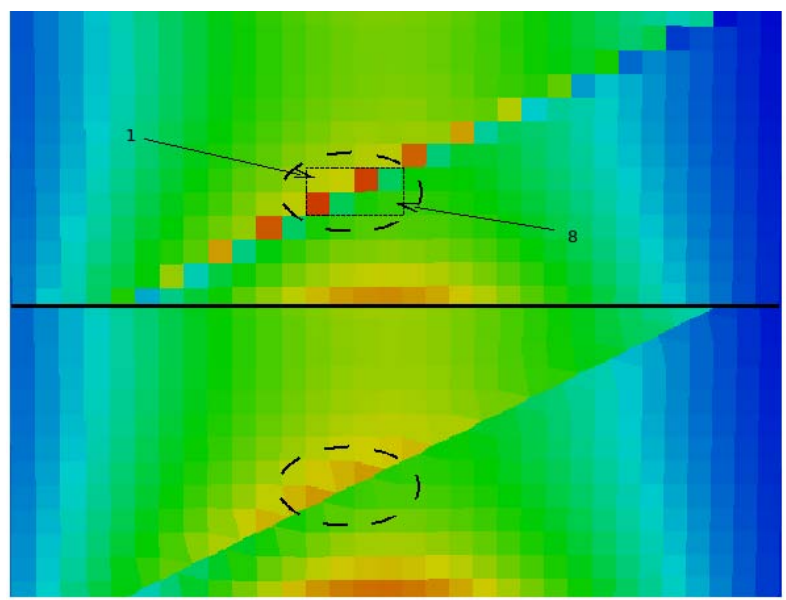

Fig. 4. Section of Figure 2 at the material interface

sphere and not along the surface where the smoothing takes place. Hence, in this situation, smoothing does not significantly affect the stresses.

In the artificial bone sample the results are similar. Smoothing only minimally affects the stresses. The elements on the surface often have unconstrained nodes or edges that are often displaced much more by smoothing than fixed nodes. However, they are not relevant for the stiffness of the model.

The changes of the von Mises stresses at the transition of the materials are analyzed by means of the sphere model, see Fig. 4 The upper material has a Young's modulus $E=5000$ and a Poisson ratio $\nu=0.3$. The lower material is more elastic with $E=12000$ and $\nu=0.1$. The transition causes a jump in the stresses. The stresses in the voxel elements at the jagged interface differ visibly in the same material as well as across the material interface. The unsmoothed model generates high peak stresses, too, that are not observed in the physical experiment. After 32 smoothing steps the interface becomes nearly a plane. The peak stress decreases from 46.8 MPa to 42.3 $\mathrm{MPa}$. The resulting stresses vary much less at the material interface, cf. Table 2

The smoothing procedure distorts elements which in turn affects the condition number of the stiffness matrix $K$. We have investigated the condition by means of two models. The first model, cube2, is obtained from cube1 by mirroring it at three faces. Thus it is 8 times bigger than cube1. The (estimated) condition numbers of the stiffness matrix $K$ after 16 smoothing steps with varying

Table 2. Stresses (in MPa) at the interface of Fig. 4. Smoothing lowers the peak stresses in the selected 8 elements by $9.6 \%$. The minium peak is increased by $24.5 \%$.

\begin{tabular}{|l|c|c|c|c|c|c|c|c|}
\hline Hexahedra & 1 & 2 & 3 & 4 & 5 & 6 & 7 & 8 \\
\hline Unsmoothed & 38.8 & 39.6 & 46.8 & 25.3 & 47.0 & 25.7 & 31.0 & 32.0 \\
\hline Smoothed & 40.1 & 41.2 & 42.3 & 31.5 & 42.5 & 31.8 & 32.9 & 33.6 \\
\hline
\end{tabular}


Table 3. Condition numbers of the stiffness matrix depending on the Euler step size $\lambda$. 16 smoothing steps were applied to the model cube2.

\begin{tabular}{|l|c|c|c|c|c|c|}
\hline smoothing step size $\lambda$ & 0.0 & 0.3 & 0.4 & 0.475 & 0.5 & 0.51 \\
\hline without preconditioning & $3.4 \cdot 10^{5}$ & $3.4 \cdot 10^{5}$ & $3.4 \cdot 10^{5}$ & $3.4 \cdot 10^{5}$ & $4.5 \cdot 10^{5}$ & - \\
\hline with ml preconditioner & 245.2 & 237.4 & 239.0 & 246.1 & 248.1 & - \\
\hline
\end{tabular}

Table 4. Condition numbers of the stiffness matrix depending on the Euler step size $\lambda$. 16 smoothing steps were applied to the 2-materials sphere.

\begin{tabular}{|l|c|c|c|c|c|}
\hline smoothing step size $\lambda$ & 0.0 & 0.5 & 0.6 & 0.67 & 0.685 \\
\hline without preconditioning & $1.75 \cdot 10^{6}$ & $1.65 \cdot 10^{6}$ & $2.31 \cdot 10^{6}$ & $1.51 \cdot 10^{7}$ & - \\
\hline with $\mathrm{ml}$ preconditioner & 507.1 & 447.0 & 486.2 & 664.5 & - \\
\hline
\end{tabular}

Euler step size $\lambda$ are given in Table 3. For either the preconditioned or the unpreconditioned system, the condition numbers are not affected much as long as $\lambda \leq 0.5$.

For the two-materials sphere corresponding numbers are found in Table 4 . Here the condition numbers vary more. Little smoothing improves the condition; too large step sizes lead to indefinite systems.

In a third test we fixed the step size $\lambda=0.4$ and varied the number of smoothing steps, cf. Table 5 . Here we observe a slow but gradual increase of the condition number up to 28 smoothing steps. Beyond this point some of the voxel elements seem to flip over causing indefinite matrices.

To investigate weak scalability we chose the artificial bone displayed in Fig. 3 that is inclosed in a cube and can be mirrored at all faces to generate arbitrarily large bones, see [2]. Not surprisingly, the computations, in particular the new smoothing and assembling procedures, show perfect weak scalability up to $216=6^{3}$ processors. Notice that all voxels are considered flexible if smoothing is applied at all. This is justified by our application, trabecular bone, where usually $3 / 4$ of the nodes are near the surface. Surprisingly, the assembling time only increased by a factor 8; apparently most of the time in this phase is due to memory accesses. Nevertheless, the assembling has become as time consuming as the solution phase. To decrease the cost of assembly we split the (distorted) hexahedra in six tetrahedra with linear basis functions. By this we regained a factor of 5 in the assembling time, however at the cost of stiffer structures, see 6].

We have tested strong scalability by means of a bone model of a fixed fracture of the distal radius with $38^{\prime} 335^{\prime} 350$ degrees of freedom. Because the patient's arm

Table 5. cube 2 model: condition numbers of the stiffness matrix depending on the number of smoothing steps with fixed $\lambda=0.4$

\begin{tabular}{|l|c|c|c|c|c|c|}
\hline \# of smoothing steps & 8 & 16 & 24 & 26 & 28 & 30 \\
\hline without preconditioner & $3.41 \cdot 10^{5}$ & $3.40 \cdot 10^{5}$ & $3.40 \cdot 10^{5}$ & $3.40 \cdot 10^{5}$ & $7.12 \cdot 10^{5}$ & - \\
\hline with ml preconditioner & 234.9 & 239.0 & 246.9 & 248.4 & 253.1 & - \\
\hline
\end{tabular}


Table 6. Fixed fracture model with 38'335'350 degrees of freedom

\begin{tabular}{|c|c|c|c|c|c|c|}
\hline & \multicolumn{3}{|c|}{ Time } & \multicolumn{3}{c|}{ Speedup } \\
\hline \#CPU & Smoothing & Assembling & Solving & Smoothing & Assembling & Solving \\
\hline 160 & 3.922 & 76.79 & 59.29 & 1 & 1 & 1 \\
\hline 240 & 2.712 & 52.44 & 38.76 & 1.45 & 1.46 & 1.53 \\
\hline 320 & 2.002 & 39.63 & 32.01 & 1.96 & 1.94 & 1.85 \\
\hline 480 & 1.382 & 26.69 & 21.22 & 2.84 & 2.87 & 2.79 \\
\hline
\end{tabular}

could not be fixed perfectly the mesh has no trabecular structure. The model consists of a full mesh. So, each node has the maximal number of neighbors and communication volume between compute nodes is relatively high. Table 6 shows the execution times and speedups for smoothing, assembling, and solving the preconditioned system. The speedups are almost linear.

\section{Conclusions}

We have parallelized a smoothing procedure originally proposed by Taubin 10 . that has been adapted by Boyd and Müller 4 for application to trabecular bones. We have observed that (1) the smoothing procedure results in a large subjective improvement of the visualization, that (2) the condition of the stiffness matrix is not increased too much as long as the elements are not distorted too severely, and that (3) the smoothing procedure applied to the model shows a reduced variation of the stresses at material transitions. However, drastically lower stresses on the surface were not obtained. Smoothing entails that the local stiffness matrices must be computed for each element which results in increased simulation times.

\section{Acknowledgments}

We acknowledge helpful discussions with Profs. Steve Boyd and Ralph Müller. The computations on the Cray XT3 have been performed in the framework of a Large User Project grant of the Swiss National Supercomputing Centre (CSCS).

\section{References}

1. Adams, M.F., et al.: Ultrascalable implicit finite element analyses in solid mechanics with over a half a billion degrees of freedom. In: ACM/IEEE Proceedings of $\mathrm{SC}(2004)$

2. Arbenz, P., et al.: A scalable multi-level preconditioner for matrix-free $\mu$-finite element analysis of human bone structures. Internat. J. Numer. Methods Engrg (to appear)

3. Arbenz, P., et al.: Multi-level $\mu$-finite element analysis for human bone structures. In: Kågström, B., et al. (eds.) PARA 2006. LNCS, vol. 4699, Springer, Heidelberg (2007) 
4. Boyd, S.K., Müller, R.: Smooth surface meshing for automated finite element model generation from 3D image data. J. Biomech. 39(7), 1287-1295 (2006)

5. Camacho, D.L.A., et al.: An improved method for finite element mesh generation of geometrically complex structures with application to the skullbase. Journal of Biomechanics 30, 1067-1070 (1997)

6. Flaig, C.: Smoothing surfaces in voxel based finite element analysis of trabecular bones. Master thesis, ETH Zurich, Inst. of Computational Science (March 2007)

7. Melton III, L.J., et al.: How many women have osteoporosis? J. Bone Miner. Res. 20(5), 886-892 (2005) Reprinted in J. Bone Miner. Res. 20(5), 886-892 (2005)

8. The PARFE Project Home Page, http://parfe.sourceforge.net/

9. Taubin, G.: A signal processing approach to fair surface design. In: SIGGRAPH 1995. Proceedings of the 22nd Annual Conference on Computer Graphics and Interactive Techniques, pp. 351-358. ACM Press, New York (1995)

10. Taubin, G.: Geometric signal processing on polygonal meshes, Eurographics State of the Art Report. 11 pages (September 2000), available from http://mesh.brown.edu/taubin/pdfs/taubin-eg00star.pdf

11. The Trilinos Project Home Page, http://software.sandia.gov/trilinos/ 